\title{
"RAPID AND SIMULTANEOUS DETERMINATION OF ADRIAMYCIN, BLEOMYCIN, VINBLASTINE AND DACARBAZINE IN PLASMA OF HODGKIN'S LYMPHOMA PATIENTS BY A REVERSED PHASE HPLC METHOD"
}

\author{
*MUHAMMAD ZUBAIR MALIK', MAHMOOD AHMAD', SALEH MUAHAMMAD \\ ${ }^{1}$ Department of Pharmacy, Faculty of Pharmacy and Alternative Medicine, \\ The Islamia University of Bahawalpur, Punjab-Pakistan \\ ${ }^{2}$ Faculty of Pharmacy, Federal Urdu University, Karachi, Sind-Pakistan \\ (Received: May 2, 2012 - Accepted: March 26, 2013)
}

\begin{abstract}
The RP-HPLC analytical method with UV detection at $230 \mathrm{~nm}$ with ODS Hypersil $\mathrm{C}_{18}$ column $(250 \mathrm{~mm} \times 4.6 \mathrm{~mm}, 5 \mu$ particle size $)$ was developed for simultaneous determination of Adriamycin, Bleomycin, Vinblastine and Dacarbazine in plasma of lymphoma patients using mobile phase composition of 300 volumes Acetonitrile and 700 volumes $0.05 \mathrm{M}$ Disodium Hydrogen Phosphate containing $0.5 \mathrm{ml}$ TEA and pH of the mobiles phase was maintained at 3.7 with $2 \mathrm{M}$ Phosphoric acid at a flow rate of $0.75 \mathrm{ml} /$ minute with linearity ranges of $0.05-50,0.06-50,0.075-50$ and $0.09-50 \mu \mathrm{g} / \mathrm{ml}$ respectively with LOD and LOQ of 0.020 , $0.050 ; 0.045,0.060 ; 0.060,0.075$ and $0.040,0.090$ respectively. \% recovery of DOX, BLM, VBL and DTIC were $99.24,99.23,99.08$ and 99.09 respectively.
\end{abstract}

Key Words: HPLC, DOX, Bleomycin, Vinblastine, Dacarbazine, Hodgkin's Lymphoma.

\section{INTRODUCTION}

Lymphoma is a type of cancer which causes extramedullary tumors arising primarily in the lymph nodes or other sites, these tumors are called lymphomas. There are two types of Lymphomas; Hodgkin's disease (HD) and Non-Hodgkin's disease (NHL) ${ }^{1}$.

This study was focused on first line treatment of Hodgkin's lymphoma i.e. ABVD (Adriamycin, Bleomycin, Vinblastine and Dacarbazine). Adriamycin also known as Doxorubicin (DOX) (IUPAC name is (7S,9S)-7-[(2R,4S,5S,6S)4-amino-5-hydroxy-6-methyloxan-2-yl]oxy-6,9,11-trihydroxy-9-(2hydroxyacetyl)-4-methoxy-8,10-dihydro-7H-tetracene-5,12-dione) is a cytotoxic anthracycline usually obtained from the fungus Streptomyces peucetius var. caesius cultures. Adriamycin is used most commonly in the treatment of Leukemia, various types of Testicular Cancer, Kaposi's sarcoma, Ewing's Sarcoma, and Hodgkin's disease ${ }^{2}$. Bleomycin (BLM) (IUPAC name is $\quad 3-\{[(2 '-\{(5 S, 8 S, 9 S, 10 R, 13 S)-15-\{6$-amino-2-[(1S)-3-amino- $1-\{[(2 S)-2,3-$ diamino-3-oxopropyl]amino $\}$-3-oxopropyl]-5-methylpyrimidin-4-yl\}-13$[\{[(2 R, 3 S, 4 S, 5 S, 6 S)-3-\{[(2 R, 3 S, 4 S, 5 R, 6 R)-4$-(carbamoyloxy)-3,5-dihydroxy6-(hydroxymethyl)tetrahydro-2H-pyran-2-yl]oxy $\}-4,5$-dihydroxy-6(hydroxylmethyl) tetrahydro-2H-pyran-2-yl]oxy $\}(1 H$-imidazol-5-yl)methyl]9-hydroxy-5-[(1R)-1-hydroxyethyl]-8,10-dimethyl-4,7,12,15-tetraoxo3,6,11,14-tetraazapentadec-1-yl\}-2,4'-bi-1,3-thiazol-4-yl)carbonyl] amino\} propyl) (dimethyl)sulfonium) is a glycopeptide antibiotic used in treatment of different types of human tumors e.g. Oesophageal Carcinoma, Cancers of head and neck, Malignant Lymphomas, Testicular Carcinoma and Malignant Pleural Effusions as it has broad-spectrum antineoplastic activity ${ }^{3}$. Vinblastine (VBL) (IUPAC name is dimethyl $(2 \beta, 3 \beta, 4 \beta, 5 \alpha, 12 \beta, 19 \alpha)-15-[(5 \mathrm{~S}, 9 \mathrm{~S})-$ 5-ethyl- 5-hydroxy- 9-(methoxycarbonyl)- $1,4,5,6,7,8,9,10$-octahydro- $2 \mathrm{H}$ 3,7-methanoazacycloundecino[5,4-b]indol- 9-yl] - 3-hydroxy- 16-methoxy1-methyl- 6,7-didehydroaspidospermidine- 3,4-dicarboxylate) is isolated from the leaves, bark, and stem of the Madagascar periwinkle (Catharanthus roseus). It is an alkaloid which is a dimmer of an Indole (Catharanthine) and a Dihydroindole (Indoline, Vindoline) moiety ${ }^{4}$. Dacarbazine (DTIC) (IUPAC name is 5-(3, 3-Dimethyl-1-triazenyl) imidazole-4-carboxamide) is used for the treatment of Hodgkin's disease, Melanoma and Soft Tissue Sarcoma, also used in combination therapies. ${ }^{5}$. The structural formulae of Adriamycin, Bleomycin, Vinblastine and Dacarbazine are shown in figure 1.
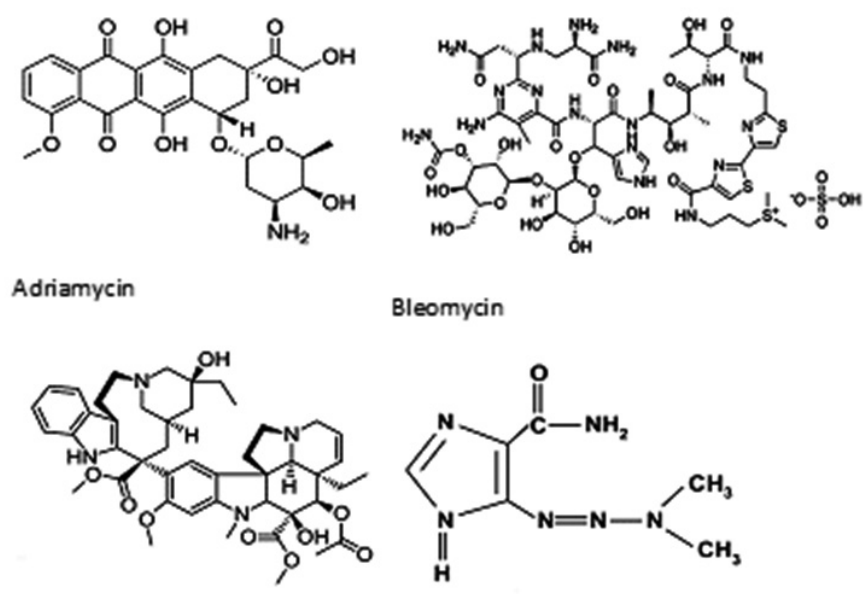

Bleomycin

Vinblastine

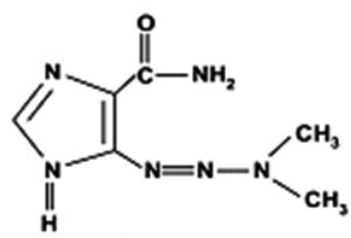

Dacarbazine

Figure 1: Structural formulae of four anticancer drugs.

An HPLC system with UV detection was used to develop a method for the simultaneous determination of ABVD. In literature, the method for simultaneous determination of ABVD using UV detector was not available. Some of the methods available for simultaneous determination of Adriamycin and 5-Flourouracil and individual determinations of ABVD are as under:

Ossama et al. (2004) introduced an HPLC method for simultaneous determination of Adriamycin hydrochloride and 5-Fluorouracil using UV detector $^{6}$. Yanhong et al. (2008) developed and validated an HPLC/Tandem Mass Spectrometric Method (LC/MS/MS) for the determination of Pegylated Liposomal Adriamycin and its metabolite Adriamycinol in the plasma of rat?

Fraier et al. (1995) developed and validated an HPLC assay for the Quantitative determination of Adriamycin in plasma and urine ${ }^{8}$. Shiu et al. (1979) introduced an HPLC method for the determination of Bleomycin $A_{2}$ and Bleomycin $\mathrm{B}_{2}$. These are two major fractions of Bleomycin Injection (Blenoxane) ${ }^{9}$. Rachid Mahdadi et al. (1991) put forward a linear-gradient, sensitive, rapid and Ion-paired Reversed Phase High Performance Liquid Chromatography Technique using fluorescence detection to quantify Bleomycin (BLM) and its metabolites in the Plasma of patients receiving Bleomycin therapy and in Hepatocytes of rat those had previously been incubated with $5 \times 10^{-5} \mathrm{M} \mathrm{BLM}^{10}$. Olaf van Tellingen et al. (1993) developed a High Performance Liquid Chromatography with Fluorescence Detection. This method was applied successfully for Pharmacokinetic studies of Vinblastine in 
mice and human. ${ }^{11}$. Peter J. Ferguson et al. (1985) used an HPLC method for analysis of possible metabolism of the Vinca Alkaloids extracts of drug-treated HL-60/CI cells. ${ }^{12}$ Stephanie L. Safgren, (2001) developed and validated a HighPerformance Liquid Chromatographic assay for simultaneous determination of Dacarbazine and its Plasma metabolites. ${ }^{13}$

The objectives of the study were the development of an HPLC method for simultaneous determination of ABVD in Plasma of Lymphoma patients and to validate the developed method according to FDA guidelines of Bioanalytical Method Validation.

\section{EXPERIMENTAL}

Disodium Hydrogen Phosphate, HPLC grade Acetonitrile, Phosphoric acid and Trichloroacetic acid were purchased from Merck, Germany and Triethylamine from Fluka, Switzerland while the reference standards of Adriamycin (India), Bleomycin (China), Vinblastine (China) and Dacarbazine (China) were obtained from Pharmedic Laboratories Pvt. Ltd., Pakistan.

Instruments

Following instruments were used during the practical work.

Hot plate magnetic stirrer (Velp Scienifca, Germany), pH meter (Inolab, Germany), Digital weighing balance (Shimadzu, AUX 220, Japan), HPLC (Agilent 1200 series, Germany), Vacuum Pump (ILMVAC, Germany), Vortex Mixer (Seouline BioScirnce, Korea), Centrifuge Machine (Helttich, Germany), Ultra sonic bath (Elma, Germany), Ultra low refrigerator (Germany).

\section{Methods}

\section{Administration of Dugs}

The anticancer drugs Adriamycin, Bleomycin, Vinblastine, and Dacarbazine were administered to the patient by the BINO (Bahawalpur Institute of Nuclear Oncology) staff through a standard procedure i.e. Bleomycin and Vinblastine were administered through Intravenous bolus injections separately. Adriamycin and Dacarbazine were administered separately in a saline/dextrose infusion depending on the condition of the patient. If the patient was diabetic then they used saline and if the patient was hypertensive then they used dextrose infusion. If the patient was both diabetic as well as hypertensive then they used Ringer Lactate.

\section{Sample Collection}

A 20-gauge venous cannula was inserted into forearm for collection of blood samples. A blood sample was collected before drug was given (zero time) and then at 5, 10, 20, 30 minutes and 1.0, 1.5, 2.0, 2.5, 3.0, 3.5, 4.0, 8.0, 12.0, 18.0, 24.0, 36.0, 48.0, 72.0, and 96.0 hours after administration of Adriamycin, Bleomycin, Vinblastine, and Dacarbazine. A $3 \mathrm{ml}$ blood sample was collected each time containing one drop of Heparin. Blood Samples were centrifuged at $5000 \mathrm{rpm}$ for 10 minutes and Plasma was collected. The Plasma samples were then frozen at $-80^{\circ} \mathrm{C}$ in the ultra low refrigerator until assay.

Method for Drug Analysis

An HPLC method was developed and standardized for the analysis of these anticancer drugs in healthy human plasma and then method was applied in Lymphoma patient's plasma successfully for pharmacokinetic evaluation of ABVD in Lymphoma patients.

Preparation of Stock Solution, Sample Solution and Blank Solution

The reference standards of Adriamycin, Bleomycin, Vinblastine and Dacarbazine were prepared by dissolving $100 \mathrm{mg}$ in $100 \mathrm{ml}$ of mobile phase separately. Further dilutions were made from these stock solutions.

$1 \mathrm{ml}$ of plasma without drugs was taken and $40 \mu \mathrm{l}$ of Trichloroacetic acid was added to precipitate the proteins and vortex for 5 minutes. It was centrifuged for 10 minutes at $5000 \mathrm{rpm}$. After centrifugation, the supernatant layer was filtered through $0.45 \mu$ membrane filter paper in a glass tube and $20 \mu 1$ was injected into the injection port of the chromatograph. The chromatogram obtained is shown in Figure 2.

$1 \mathrm{ml}$ of plasma sample of Hodgkin's Lymphoma Patient treated with Adriamycin, Bleomycin, Vinblastine and Dacarbazine was taken and added $40 \mu$ of Trichloroacetic acid to precipitate the proteins and vortex for 5 minutes. It was centrifuged for 10 minutes at $5000 \mathrm{rpm}$. After centrifugation, the clear supernatant layer was filtered through $0.45 \mu$ filter in a glass tube and $20 \mu 1$ was injected into the injection port of the chromatograph. The chromatogram obtained is shown in Figure 3.

Time

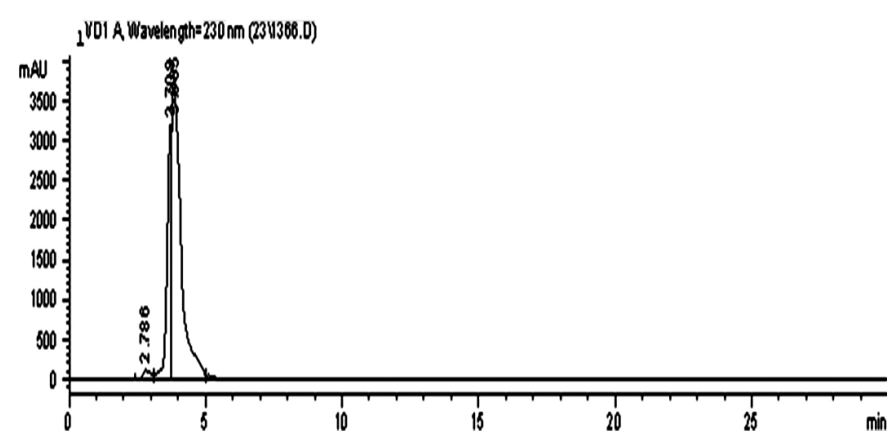

Figure 2: Chromatogram of Blank Plasma of Hodgkin's Lymphoma Patient before administration of four anticancer drugs.

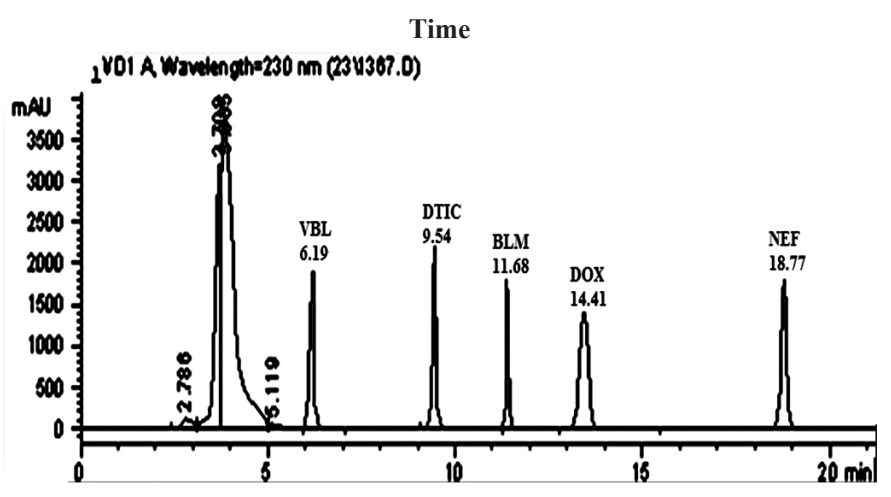

Figure 3: Chromatogram of Plasma Sample of Hodgkin's Lymphoma Patient treated with Adriamycin, Bleomycin, Vinblastine and Dacarbazine.

\section{Preparation of Standard Curve}

From the four stock solutions of working standards, a range of 0.098 , $0.195,0.391,0.781,1.562,3.125,6.25,12.5,25$ and $50 \mu \mathrm{g} / \mathrm{ml}$ of combined reference standards solutions containing each of Adriamycin, Bleomycin, Vinblastine and Dacarbazine was prepared.

To $1 \mathrm{ml}$ of blank plasma, was added $100 \mu \mathrm{l}$ of combined working standards solutions at concentration range of $0.098-50 \mu \mathrm{g} / \mathrm{ml}$ of each of Adriamycin, Bleomycin, Vinblastine and Dacarbazine.

To the resulting solutions were added $20 \mu \mathrm{l}$ of Trichloroacetic acid to precipitate the proteins and vortex for 5 minutes and centrifuged at $5000 \mathrm{rpm}$ for 10 minutes. The supernatant layer was filtered through $0.45 \mu$ membrane filter paper in a glass tube and $20 \mu 1$ of this filtered standard solution was injected into the chromatograph. Nefopam was used as the internal standard $10 \mu \mathrm{g} / \mathrm{ml}$.

\section{High Performance Liquid Chromatography}

An Agilent 1200 series Chromatograph consisted of Isocratic Pump with UV-Detector, column with dimensions $4.6 \mathrm{~mm} \mathrm{X} 250 \mathrm{~mm}$ and computer software to handle it was used. The flow rate was $0.75 \mathrm{ml} / \mathrm{min}$. The UV detection of Adriamycin, Bleomycin, Vinblastine and Dacarbazine was set at $230 \mathrm{~nm}$. The mobile phase consisted of 300 volumes Acetonitrile and 700 volumes $0.05 \mathrm{M}$ Disodium Hydrogen Phosphate containing $0.5 \mathrm{ml}$ TEA and $\mathrm{pH}$ of the mobiles phase was maintained at 3.7 with $2 \mathrm{M}$ Phosphoric acid.

The Mobile Phase was prepared by dissolving $8.9 \mathrm{~g}$ i.e. $0.05 \mathrm{M}$ Disodium Hydrogen Phosphate $\mathrm{Na}_{2} \mathrm{HPO}_{4}$ in $200 \mathrm{ml}$ of HPLC water and sonicated to dissolve completely and then made the volume up to $1000 \mathrm{ml}$ with HPLC water. Mixed thoroughly and $700 \mathrm{ml}$ of it was taken and $300 \mathrm{ml}$ of Acetonitrile HPLC grade in a $1 \mathrm{~L}$ beaker added $0.5 \mathrm{ml}$ TEA and mixed completely on a magnetic stirrer. Then adjusted the $\mathrm{pH}$ of the mobile phase to 3.7 using 2M Phosphoric acid.

Plasma Spiking and Extraction Procedure

The plasma was spiked with reference standards containing $0.098,25$ and $50 \mu \mathrm{g} / \mathrm{ml}$ of each of Adriamycin, Bleomycin, Vinblastine and Dacarbazine. The compounds were recovered by precipitating the plasma with $20 \mu \mathrm{l}$ Trichloroacetic Acid and centrifuging at $5000 \mathrm{rpm}$ for 10 minutes and then 
injecting the supernatant into the chromatograph.

Validation of Method

Validation of method was done according to FDA guidelines of Bioanalytical Method validation. Following performance characteristics of bioanalytical method validation were studied; accuracy, precision (Intra day and inter day), recovery, linearity, limit of detection and limit of quantitation.

In the development process, different compositions of mobile phase with several combinations of buffer and organic phase were tested. Acetonitrile, Methanol and Buffer in varying percentages were tested and final mobile phase having composition of Disodium Phosphate-Acetonitrile in the molar ratio $70: 30(\% \mathrm{v} / \mathrm{v})$ and $0.05 \%$ Triethylamine (TEA) which provided high resolution and separation with sharp peaks for Adriamycin, Bleomycin, Vinblastine and Dacarbazine (ABVD). The $\mathrm{pH}$ and molarity of the mobile phase was selected after different trials. The $\mathrm{pH} 3.7$ adjusted with $2 \mathrm{M}$ Phosphoric acid was found suitable for the desired objectives of not absorbing at low wavelength and was sufficient in concentration to avoid peak tailing because silica based particles are unstable at low $\mathrm{pH}(\mathrm{pH}<2)$. The experimentation was also performed for the determination of optimal flow rate for pumping the mobile phase in HPLC system. Flow rate of $0.75 \mathrm{ml} / \mathrm{min}$. was found appropriate for peak resolution with short retention time compared with available methods.

Similarly, the stationary phase of suitable dimensions was also selected for providing added benefits of retention and separation. The run time was short requiring only 20 minutes. The retention times for Adriamycin, Bleomycin, Vinblastine, Dacarbazine and internal standard Nefopam were 14.41, 11.68, $6.19,9.54$ and $18.77 \mathrm{~min}$., respectively. Linearity of the assay method was determined by constructing calibration (standard) curve to find out the relationship between instrument response and known concentrations of the analytes. The number of standards used in constructing a calibration curve was selected to the anticipated range of analytical values of ABVD combination for lymphoma cancer patient keeping in mind the concentration of drugs in plasma and nature of analyte-response relationship. The calibration curves were prepared in the same biological matrix (plasma) by spiking the plasma matrix with known concentrations of the analytes. Each drug concentration in three replicates was run in the HPLC system (Agilent-Technologies 1200 series) and the data was plotted to calculate the parameters of standard curve. The values of these parameters were found to be consistent with FDA guidelines of method validation. Results are shown in Table 1.

The accuracy of an analytical method describes the closeness of mean test results obtained by true value (concentration) of the Analyte. Accuracy is determined by triplicate analysis of low, medium and high concentrations of samples containing known amounts of the analyte. The Accuracy in present method is measured by using a minimum of three concentrations in the range of expected concentrations (intra-day). The deviation of mean from true value serves as the measure of accuracy. These values were found in the range of described by FDA for bioanalytical methods. The precision of an analytical method describes the closeness of individual measures of the Analyte when the procedure is applied repeatedly to multiple aliquots of a single homogenous volume of biological fluid (inter-day). The Precision was measured in triplicates with three concentrations in the range of expected concentrations. The percent coefficient of variation $(\% \mathrm{CV})$ in both intra-day and inter-day was less than $2 \%$ which is well within the range of $15 \%$ for lowest and $20 \%$ for highest concentration of FDA criteria for biological fluids. The results are given in Table 2 and Table $3 .^{14}$

The Extraction yield (recovery) of an analyte in an analysis method is the detector response obtained from an amount of the analyte added to and extracted from the plasma, compared to detector response obtained for known concentration of sample. In current method, the extraction efficiency found to be consistent, precise, and reproducible. The Recovery experiments were performed by comparing the analytical results for extracted samples at three concentration levels with un-extracted standards that represent $100 \%$ recovery. The extraction yields found in the present method were better than previously published methods. The results of \%recovery are given in Table 4. The present method was successfully applied for the assay of all samples of current study. The method was found to be simple, sensitive, and stable. The Plasma concentrations of each sample were computed by measuring peak areas of each sample with the peak areas of standard curve. The plasma concentrations were plotted on rectangular co-ordinate graphs.

\section{Safety Analysis}

The Health assessment including vital signs, physical examination and clinical laboratory testing including complete blood count was performed before administration of anticancer regimen. Test reports were checked one day before administration of the anticancer regimen.

Table 1: Regression and statistical parameters for determination of ABVD
(Adriamycin, Bleomycin, Vinblastine and Dacarbazine) by the proposed HPLC method.

Table 2: Results of the Intra day precision and accuracy studies for

\begin{tabular}{|c|c|c|c|c|c|c|c|c|}
\hline \multirow[t]{2}{*}{$\begin{array}{l}\text { Linearity } \\
\text { Range } \\
(\mu \mathrm{g} / \mathrm{ml})\end{array}$} & \multicolumn{3}{|c|}{$\underline{\text { Regression data }}$} & \multirow[t]{2}{*}{$\mathrm{S} / \mathrm{Y}^{\mathrm{d}}$} & \multirow[t]{2}{*}{$\mathrm{S}_{\mathrm{a}} \mathrm{e}^{\mathrm{e}}$} & \multirow[t]{2}{*}{$\mathrm{S}_{\mathrm{b}} \mathrm{f}$} & \multirow{2}{*}{$\begin{array}{l}\text { LOD g } \\
(u g / m l)\end{array}$} & \multirow{2}{*}{$\begin{array}{l}\mathrm{LOQ}^{\mathrm{h}} \\
(\mu \mathrm{g} / \mathrm{ml}\end{array}$} \\
\hline & $\mathrm{a}^{\mathrm{a}}$ & $\mathrm{b}^{\mathrm{b}}$ & $\mathrm{r}^{\mathrm{c}}$ & & & & & \\
\hline $\begin{array}{l}\text { Adriamycin } \\
(0.05-50)\end{array}$ & 22.4 & 21.2 & 0.995 & 0.002 & 0.3 & 0.4 & 0.020 & 0.050 \\
\hline $\begin{array}{l}\text { Bleomycin } \\
(0.06-50)\end{array}$ & 71.9 & 79.6 & 0.998 & 0.003 & 1.0 & 0.7 & 0.045 & 0.060 \\
\hline $\begin{array}{l}\text { Vinblastine } \\
(0.75-50)\end{array}$ & 65.6 & 43.3 & 0.994 & 0.002 & 0.5 & 0.7 & 0.060 & 0.075 \\
\hline $\begin{array}{l}\text { Dacarbazine } \\
(0.09-50)\end{array}$ & 22.1 & 4.4 & 0.996 & 0.001 & 0.01 & 0.3 & 0.040 & 0.090 \\
\hline 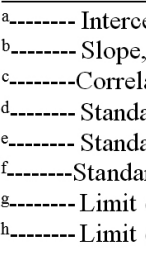 & $\begin{array}{l}\text { tion co } \\
\text { rd devi } \\
\text { rd devi } \\
\text { d devi } \\
\text { of detec }\end{array}$ & $\begin{array}{l}\text { efficie } \\
\text { ation o } \\
\text { ation } \\
\text { tion o } \\
\text { tion, }\end{array}$ & $\begin{array}{l}\text { t, } \\
\text { residua } \\
\text { interce, } \\
\text { slope, }\end{array}$ & & & & & \\
\hline
\end{tabular}

Adriamycin (DOX), Bleomycin (BLM), Vinblastine (VBL) and Dacarbazine (DTIC).

Table 3: \% Recovery of ABVD, Adriamycin (DOX), Bleomycin (BLM), Intra day \% Precision

\begin{tabular}{|c|c|c|c|}
\hline \multirow[b]{2}{*}{ Compound } & \multirow[b]{2}{*}{$98 \mathrm{ng} / \mathrm{ml}$} & \multicolumn{2}{|c|}{$\mu \mathrm{g} / \mathrm{ml}($ mean \pm S. D. $)$} \\
\hline & & 25 & 50 \\
\hline DOX & $97.88 \pm 0.62$ & $24.79 \pm 0.075$ & 49.910 .083 \\
\hline BLM & $97.59 \pm 0.72$ & $24.84 \pm 0.105$ & 49.930 .028 \\
\hline VBL & $97.74 \pm 0.77$ & $24.87 \pm 0.071$ & 49.730 .236 \\
\hline DTIC & $97.77 \pm 0.72$ & $24.74 \pm 0.071$ & 49.500 .162 \\
\hline \multicolumn{4}{|c|}{ Intra day $\%$ Accuracy } \\
\hline & & \multicolumn{2}{|l|}{$\mu \mathrm{g} / \mathrm{ml}$ (mean) } \\
\hline Compound & $98 \mathrm{ng} / \mathrm{ml}$ & 25 & 50 \\
\hline DOX & $99.88 \pm 0.82$ & $99.19 \pm 0.72$ & $99.82 \pm 0.45$ \\
\hline BLM & $99.58 \pm 0.74$ & $99.39 \pm 0.78$ & $99.86 \pm 0.76$ \\
\hline VBL & $99.74 \pm 0.66$ & $99.50 \pm 0.65$ & $99.47 \pm 0.87$ \\
\hline DTIC & $98.67 \pm 0.52$ & $99.50 \pm 0.70$ & $99.01 \pm 0.90$ \\
\hline
\end{tabular}

Vinblastine (VBL) and Dacarbazine (DTIC) from the spiked plasma by the proposed HPLC method.

Table 4: Results of the Inter day precision and accuracy studies for

\begin{tabular}{|c|c|c|c|c|c|c|c|}
\hline \multicolumn{4}{|c|}{$\begin{array}{l}\text { Spiked plasma conc. } \\
(\mu \mathrm{g} / \mathrm{ml})\end{array}$} & \multicolumn{4}{|c|}{$\%$ Recovery $^{a}$} \\
\hline $\mathrm{DOX}$ & BLM & VBL & DTIC & DOX & BLM & VBL & DTIC \\
\hline 0.098 & 0.098 & 0.098 & 0.098 & 99.29 & 99.29 & 99.35 & 99.44 \\
\hline 25 & 25 & 25 & 25 & 99.19 & 99.51 & 99.10 & 99.21 \\
\hline$\underline{50}$ & 50 & 50 & 50 & 99.24 & 99.23 & 99.08 & 99.09 \\
\hline
\end{tabular}


Adriamycin (DOX), Bleomycin (BLM), Vinblastine (VBL) and Dacarbazine (DTIC).

\section{RESULTS AND DISCUSSION}

\begin{tabular}{lccc}
\hline & \multicolumn{3}{c}{ Inter day Precision } \\
\cline { 3 - 4 } Compound & $98 \mathrm{ng} / \mathrm{ml}$ & 25 & 50 \\
\hline DOX & $97.77 \pm 0.63$ & $24.85 \pm 0.101$ & $49.88 \pm 0.11$ \\
BLM & $97.70 \pm 0.43$ & $24.78 \pm 0.100$ & $49.65 \pm 111$ \\
VBL & $97.65 \pm 0.63$ & $24.81 \pm 0.088$ & $49.93 \pm .045$ \\
DTIC & $97.70 \pm 0.43$ & $24.80 \pm 0.116$ & $49.83 \pm 0.142$
\end{tabular}

\begin{tabular}{lccc}
\hline \multicolumn{3}{c}{ Inter day \% Accuracy } \\
\cline { 3 - 4 } & & \multicolumn{2}{c}{$\mu \mathrm{g} / \mathrm{ml}$ (mean) } \\
\cline { 3 - 4 } Compound & $98 \mathrm{ng} / \mathrm{ml}$ & 25 & 50 \\
\hline DOX & $96.77 \pm 0.45$ & $98.40 \pm 0.34$ & $99.76 \pm 0.55$ \\
BLM & $98.69 \pm 0.40$ & $98.11 \pm 0.89$ & $99.30 \pm 0.88$ \\
VBL & $97.64 \pm 0.78$ & $98.25 \pm 0.63$ & $99.85 \pm 0.75$ \\
DTIC & $98.10 \pm 0.56$ & $99.22 \pm 0.23$ & $99.66 \pm 0.65$
\end{tabular}

The standard curves of ABVD (Adriamycin, Bleomycin, Vinblastine, and Dacarbazine) were constructed using known plasma concentrations. The ranges of these samples were $98 \mathrm{ng} / \mathrm{ml}$ to $50 \mu \mathrm{g} / \mathrm{ml}$ for the four drugs which covered the individual ranges of four drugs in the plasma. The Linearity regression was applied to fit straight line. The results of standard curve parameters are presented in Table 1, 2, 3, and Table 4. The FDA guidelines were followed for the development and validation of the analytical method. The Plasma calibration curves were constructed and assayed in triplicates on three different periods to evaluate linearity, precision, accuracy, and percent extraction yield/ percent recovery, limit of quantitation, limit of detection, selectivity and stability.

In current method, stability of the Analyte was determined after three freeze and thaw cycles. Three aliquots at each of the low, medium, and high concentrations were stored at $-80^{\circ} \mathrm{C}$ for 24 hours and thawed at room temperature. When completely thawed, the samples were refrozen for 24 hours under the same conditions. The freeze-thaw cycle was repeated for three times, analyzed on each cycle for the determination of drugs in each aliquot. The method showed its stability for four drugs (ABVD). The values were found in acceptable range of stability. The value of Percent Difference was less than 3 for all concentrations included for stability testing. The method was found to be stable for testing this drug in human plasma.

Qingyu et al. (2002) reported within-day and between day \%CV were less than $13 \%[15]$ while present study found percent difference less than $3 \%$ which is better than the values reported by Qingyu et al. (2002).

\section{CONCLUSION}

The HPLC analytical method developed was simple and fast for the simultaneous determination of four anticancer drugs (Adriamycin, Bleomycin, Vinblastine and Dacarbazine) used in the treatment of Lymphoma cancer.

\section{ACKNOWLEDGEMENTS}

The authors are highly thankful to kind support of the Director BINO (Bahawalpur Institute of Nuclear Oncology) hospital Prof. Dr. Naeem Laghari for providing the Hodgkin's Lymphoma patients. The authors are also obliged to the management of Pharmedic Pharmaceutical Pvt. Ltd. for providing the reference standards of Adriamycin, Bleomycin, Vinblastine and Dacarbazine.
1. A. Helms Richard (2006): Textbook of Therapeutics, Drug and Disease Management, Eighth Edition.

2. R. Rodney Larson, M. B. Khazaeli, and H. Kenneth Dillon (2003): Development of an HPLC method for simultaneous analysis of Five Antineoplastic Agents. Applied Occupational and Environmental Hygiene. 18(2): 109-119.

3. M. Mohamed Sayed-Ahmed, Ph.D., H. Heba Mansour Ph.D., A. Ola Gharib, Ph.D., and Hafez F. Hafez, M.D. (2004): Acetyl-L-Carnitine Modulates Bleomycin-Induced OxidativenStress and Energy Depletion in Lung Tissues, Journal of the Egyptian National Cancer Institute. 16(4): 237-243.

4. W.A. Creasey, , A.I. Scott, C.C. Wei, J. Kutcher, A. Schwartz, and J.C. Marsh (1975): Pharmacological studies with vinblastine in the dog. Cancer Research 35:1116-1120.

5. L. Stephanie Safgren, M. Joel Reid, Robert Rios, M. Matthew Ames. (2001): Validated high-performance liquid chromatographic assay for simultaneous determination of dacarbazine and the plasma metabolites 5-(3-hydroxymethyl-3-methyl-1-triazeno) imidazole-4- carboxamide and 5-(3-methyl-1-triazeno) imidazole-4-carboxamide Journal of Chromatography B. 754: 91-96.

6. T. Ossama Fahmy, A. Mohamed Korany, M. Hadir Maher (2004): High performance liquid chromatographic determination of some coadministered anticancer drugs in pharmaceutical preparations and in spiked human plasma. Journal of Pharmaceutical and Biomedical Analysis. 34: 1099-1107.

7. Yanhong Liu, Yuhui Yang, Xiangtao Liu, Tao Jiang. (2008): Quantification of pegylated liposomal doxorubicin and doxorubicinol in rat plasma by liquid chromatography/electrospray tandem mass spectroscopy: Application to preclinical pharmacokinetic studies. Talanta. 74: 887-895.

8. D. Fraier, E. Frigerio, E. Pianezzola, M. Strolin benedetti, J. Cassidy and P. Vasey (1995): A sensitive procedure for the quantitation of free and N-(2-hydroxypropyl) methacrylamide polymer-bound doxorubicin (PK1) and some of its metabolites, 13-dihydrodoxorubicin, 13-dihydrodoxorubicinone and doxorubicinone, in human plasma and urine by reversed-phase HPLC with fluorimetric detection,/ Journal of Pharmaceutical \& Biomedical Analysis. 13(4/5): 625-633.

9. G.K. Shiu, T.J. Goehl, and W.H. Pitlick (1979): A rapid high-performance liquid chromatographic (HPLC) for the detection of bleomycin $\mathrm{A}_{2}$ and $\mathrm{B}_{2}$ in human plasma. Journal of Pharmaceutical Sciences. 68: 232-4.

10. Rachid Mahdadil, Abdelraouf Kenani 2, Nieole Pommeryl, Jean Pommeryl, Jean Pierre H6nichart2, and Michel Lhermitte. (1991): Highperformance liquid chromatography assay of bleomycin in human plasma and rat hepatocytes in culture, Cancer Chemotherapy Pharmacology. 28:22-26

11. Olaf van Tellingen, H. Jos Beijnen, J. Willem Nooijen, and Auke Bult. (1993): Plasma Pharmacokinetics of Vinblastine and the Investigational Vinca Alkaloid W-(Deacetyl-0-4-vinblastoyl-23)-L-ethyl Isoleucinate in Mice as Determined by High-Performance Liquid Chromatography, Cancer Research. 53: 2061-2065.

12. J. Peter Ferguson and E. Carol Cass (1985): Differential Cellular Retention of Vincristine and Vinblastine by Cultured Human Promyelocytic Leukemia HL-60/CI Cells: The Basis of Differential Toxicity. Cancer Research. 45: 5480-5488.

13. D. Murry, and R. Classon, (2004): LC/MS Method for the Determination of Vinblastine and Vincristine in Human Plasma. AAPS Journal Pharmaceutical Sciences 5:81.

14. FDA Guidelines, "Guidance for Industry, Bioanalytical Method Validation". http://www.fda.gov./cder/guidance/index.htm.

15. Qingyu Zhou, Balram Chowbay. (2002): Determination of doxorubicin and its metabolites in rat serum and bile by LC: application to preclinical pharmacokinetic studies. Journal of Pharmaceutical and Biomedical Analysis. 30: 1063-1074. 